\title{
Combining target enrichment with barcode multiplexing for high throughput SNP discovery
}

\author{
Nik Cummings ${ }^{1}$, Rob King ${ }^{5}$, Andre Rickers ${ }^{5}$, Antony Kaspi ${ }^{2}$, Sebastian Lunke ${ }^{3}$, Izhak Haviv ${ }^{2,4,6,7}$, \\ Jeremy BM Jowett ${ }^{1,4^{*}}$
}

\begin{abstract}
Background: The primary goal of genetic linkage analysis is to identify genes affecting a phenotypic trait. After localisation of the linkage region, efficient genetic dissection of the disease linked loci requires that functional variants are identified across the loci. These functional variations are difficult to detect due to extent of genetic diversity and, to date, incomplete cataloguing of the large number of variants present both within and between populations. Massively parallel sequencing platforms offer unprecedented capacity for variant discovery, however the number of samples analysed are still limited by cost per sample. Some progress has been made in reducing the cost of resequencing using either multiplexing methodologies or through the utilisation of targeted enrichment technologies which provide the ability to resequence genomic areas of interest rather that full genome sequencing.
\end{abstract}

Results: We developed a method that combines current multiplexing methodologies with a solution-based target enrichment method to further reduce the cost of resequencing where region-specific sequencing is required. Our multiplex/enrichment strategy produced high quality data with nominal reduction of sequencing depth. We undertook a genotyping study and were successful in the discovery of novel SNP alleles in all samples at uniplex, duplex and pentaplex levels.

Conclusion: Our work describes the successful combination of a targeted enrichment method and index barcode multiplexing to reduce costs, time and labour associated with processing large sample sets. Furthermore, we have shown that the sequencing depth obtained is adequate for credible SNP genotyping analysis at uniplex, duplex and pentaplex levels.

\section{Background}

The development of massively parallel sequencing or next generation sequencing (NGS) platforms provide the capacity for high-throughput sequencing of whole genomes at low cost. However, while those platforms improve the capacity to find novel variations that are not covered by existing genotyping arrays, they do not make use of the existing data, composed of thousands of relatively small genomic regions that have been associated with diseases through the use of genome wide association and linkage studies, where isolation of causative genetic variants has been problematic.

\footnotetext{
* Correspondence: Jeremy.Jowett@bakeridi.edu.au

${ }^{1}$ Genomics and Systems Biology, Baker IDI Heart and Diabetes Institute, Melbourne, Australia

Full list of author information is available at the end of the article
}

The efficiency of NGS-mediated genotyping has recently been improved through employing amplicon libraries of long-range PCR, which encompass discrete genomic intervals [1]. However, this method of library construction remains time-consuming, costly and limited to very small genomic regions ( $5 \mathrm{kbp}-1 \mathrm{Mbp}$ ) and is impractical for genetic dissection of disease linked loci which can span $10 \mathrm{Mb}$ or more. The development of molecular inversion probes (MIPs) and the use of chipbased technologies for massively parallel capture of specific genomic targets is limited by representational and allelic bias and remains costly and time consuming $[2,3]$. Recent advances in genome enrichment technologies provide efficient methods of region-specific, in-solution partitioning of regions spanning several megabases $[4,5]$. These technologies can be used to "capture" whole contiguous regions or generate exon specific libraries for
C Biomed Central

(c) 2010 Cummings et al; licensee BioMed Central Ltd. This is an Open Access article distributed under the terms of the Creative Commons Attribution License (http://creativecommons.org/licenses/by/2.0), which permits unrestricted use, distribution, and reproduction in any medium, provided the original work is properly cited. 
discovery of functional variants within regions determined by genome wide association and linkage studies. However, the efficiency of genome partitioning and NGS is also compromised, since at the current size of capture for example up to $10 \mathrm{MB}$, the depth of read for a single sample, through a single lane of Illumina GAIIx (50-170 fold coverage), far exceeds that needed for confident SNP calling.

Nucleotide-based barcodes have been used to multiplex individual samples for use on NGS platforms [1,6-9]. This methodology exploits the sequencing depth of NGS technologies to sequence multiple samples in a single flow cell, reducing costs and increasing throughput. While these methodologies represent a significant advance in resequencing throughput, they do not provide the ability to target sequencing to specific diseaselinked loci. The importance of continued sequencing efforts, particularly in focused populations, to analyse the differences between disease-affected and unaffected individuals has been recognised [10]. To achieve this outcome, the next logical step has been to combine multiplexing barcode technology with targeted enrichment. This has the effect of focussing the power of NGS sequencing onto a particular region (which contains putative disease genes), and simultaneously allowing pooling and later de-convolution of individual DNA samples. The outcome of this is to increase the throughput and significantly reduce the cost of resequencing disease-linked loci in large cohorts.

Our experimental data describes a cost effective, high throughput method for region-specific, multiplex sequencing by combining genome partitioning and barcode indexing with NGS technology. Individual samples were multiplexed prior to sequencing and successfully separated in silico after sequencing on the Illumina GAII platform. Genomic libraries were selectively enriched for the human chromosome X exome using Agilent's SureSelect methodology and resulting sequences were efficiently mapped to the human $\mathrm{X}$ chromosome.

\section{Results}

Eight genomic DNA samples from 5 individuals (labelled A1, A2, A3, B1, B2, C1, D1 and E1) were used. All samples were processed in the same manner except sample A1, which was not indexed, but was partitioned and sequenced as a uniplex. Samples A2 and B1 were indexed, pooled, partitioned and sequenced as a duplex. Samples A3, B2, C1, D1 and E1 were indexed, pooled, partitioned and sequenced as a pentaplex. Samples A, B and E were taken from male participants and samples $C$ and $D$ were from female participants to allow for the influence of chromosome $\mathrm{X}$ copy number on partitioning results. This design allows us to determine (1) the efficiency of deconvoluting individual samples from a multiplex at duplex and pentaplex levels (2) sequence consistency between uniplex and multiplex samples and (3) the effect of multiplex indexing on target enrichment. A schematic representation of sample preparation is shown in Figure 1A. Barcode indexing was performed with indexing primers from Illumina (Illumina, California, USA) and GeneWorks (GeneWorks, Adelaide, Australia) and genome partitioning was performed using SureSelect Human Chromosome X Exome Kit (Agilent, California, USA).

To test the feasibility and utility of multiplexing individuals for genotyping through genomic partitioning and NGS, we compared the genotyping data of the same individual, in the context of uniplex, duplex, and pentaplex genomic mixes. We prepared genomic DNA from white blood cells of five individuals, fragmented the DNA, and constructed three adaptor ligated libraries, each for one Illumina GAII lane. In one lane, the individual was genotyped as uniplex, in the second, as duplex with another individual, and in the third lane, as pentaplex with four additional individuals. The single individual genomes (total of eight) were tracked through the inclusion of six mer indices that is unique to each individual.

Sequence generation and processing was performed on the Illumina GAII platform using Illumina pipeline $(\mathrm{v}$ 1.3.2). Sequencing was performed as $65 \mathrm{bp}$ single-end reads. Sequences were aligned to the hg18 reference genome using the BWA aligner (v 0.5.5) [11]. Resulting files were modified with the Picard toolkit [12] before SNP analysis was undertaken using Genome Analysis Toolkit (GATK). A comprehensive description of library preparation, target enrichment and bioinformatics is provided in the Materials and Methods section of this paper.

Analysis of the uniplex data (sample A1) shows $16,403,360$ unique sequences across the human genome. Of these, $26 \%$ of sequences were mapped to the X chromosome. The baits for target enrichment were designed for exons within the $\mathrm{X}$ chromosome totalling 3.3 Mb. $88 \%$ of sequence reads map on or within 500 bp of targeted exons. This represents 97 -fold enrichment of the $\mathrm{X}$ chromosome exons (Table 1). An example of tiled sequence aligning to $\mathrm{X}$ chromosome exons is shown in Figure $1 \mathrm{~B}$. The genome browser clearly shows the vast over-representation of reads from the genome that span the target areas and low "background" reads outside of these areas. 2,399 SNPs were discovered on the X chromosome, 1,839 (77\%) of these were found to be previously annotated on dbSNP and 560 were novel SNPs (Table 2). We used a GATK confidence parameter of 70 , which minimises the discovery of the false positives, yet retains identification of novel SNPs. The parameter was chosen by comparing the converging plots of total number of SNPs detected and the proportion of these annotated on dbSNP (Figure 1D). With increasing stringency the two plots tend toward parallel with the 


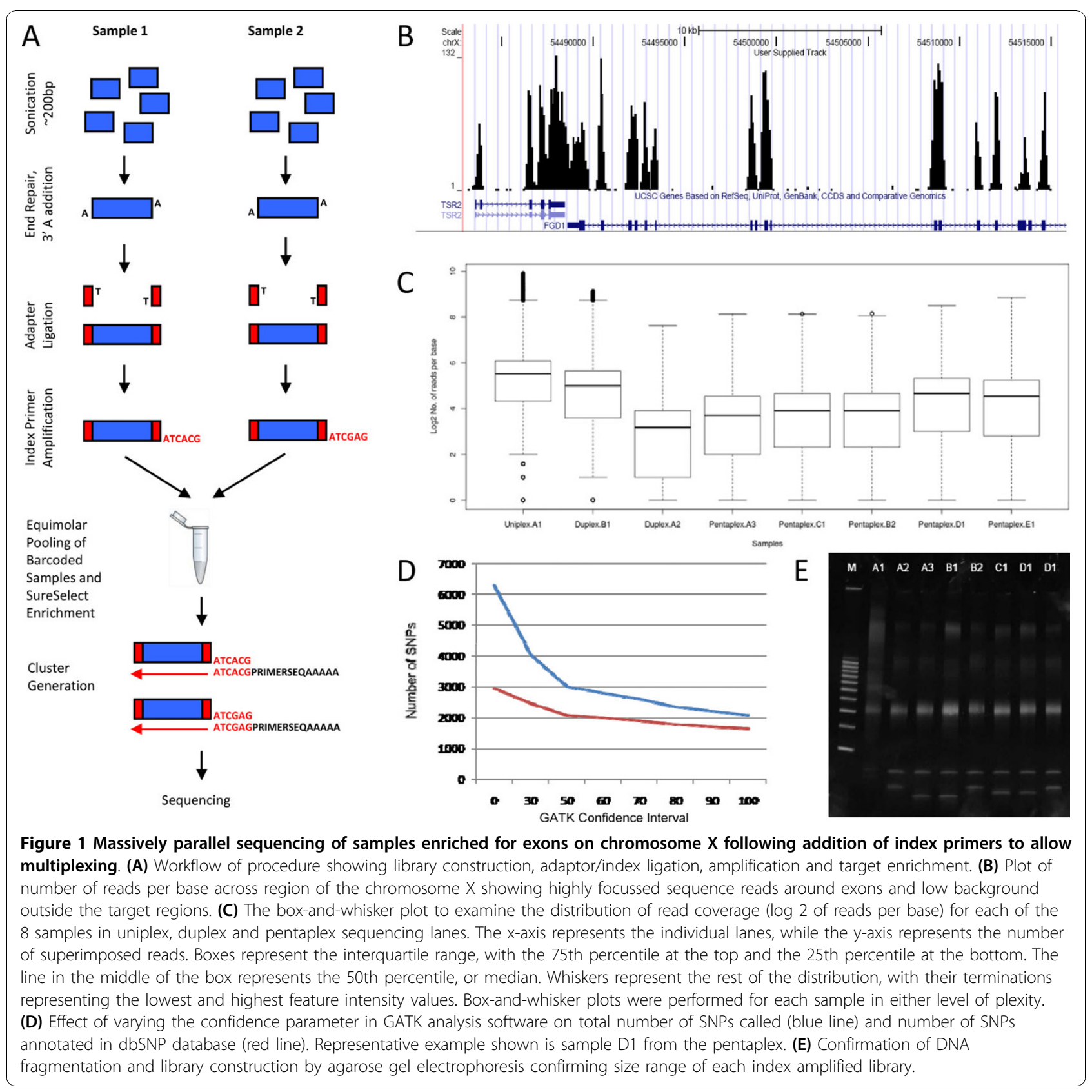

Table 1 Sequencing and target enrichment results for 8 samples in uniplex, duplex and pentaplex reactions

\begin{tabular}{|c|c|c|c|c|c|c|c|}
\hline Sample & Plexity & Filtered Reads & Mapped to ChrX & Mapped to Target Region & \% Mapped to X & $\%$ Mapped to Target & Fold Enrichment \\
\hline A1 & Uniplex & $16,403,360$ & $4,044,511$ & $3,564,834$ & 25.6 & 22.5 & 97 \\
\hline$A 2$ & Duplex & $2,035,467$ & 730,156 & 666,572 & 36.9 & 33.7 & 170 \\
\hline B1 & Duplex & $11,833,641$ & $2,655,666$ & $2,313,476$ & 23.2 & 20.2 & 85 \\
\hline A3 & Pentaplex & $3,037,781$ & $1,131,596$ & $1,036,600$ & 38.1 & 34.9 & 180 \\
\hline B2 & Pentaplex & $3,204,352$ & $1,230,630$ & $1,129,857$ & 39.3 & 36.0 & 190 \\
\hline $\mathrm{C} 1$ & Pentaplex & $3,122,661$ & $1,220,017$ & $1,123,547$ & 40.1 & 36.9 & 196 \\
\hline D1 & Pentaplex & $3,687,146$ & $1,944,667$ & $1,778,223$ & 53.8 & 49.2 & 324 \\
\hline E1 & Pentaplex & $3,078,897$ & $1,832,550$ & $1,696,950$ & 60.6 & 56.1 & 427 \\
\hline
\end{tabular}


Table 2 Variant identification results across target region by sample

\begin{tabular}{lrrrrr}
\hline Sample & Plexity & Total SNP* & Annotated & Novel & \% Annotated \\
\hline A1 & Uniplex & 2,399 & 1,839 & 560 & $77 \%$ \\
A2 & Duplex & 1,462 & 991 & 471 & $68 \%$ \\
B1 & Duplex & 2,385 & 1,627 & 758 & $68 \%$ \\
A3 & Pentaplex & 2,102 & 1,242 & 860 & $59 \%$ \\
B2 & Pentaplex & 1,910 & 1,289 & 621 & $67 \%$ \\
C1 & Pentaplex & 2,033 & 1,362 & 671 & $67 \%$ \\
D1 & Pentaplex & 2,600 & 1,904 & 696 & $73 \%$ \\
E1 & Pentaplex & 2,390 & 1,760 & 630 & $74 \%$ \\
\hline
\end{tabular}

* Calculations performed using confidence parameter of 70 in GATK.

difference comprising novel SNPs. All plots were similar, a representative plot is shown (sample D1 from pentaplex).

Duplex data (samples A2 and B1) were split by index barcode and analysed independently. For sample A2, there were 2,035,467 unique sequences across the human genome. Of these, $37 \%$ of sequences were mapped to the X chromosome, of these $91 \%$ of sequence reads map on or within $500 \mathrm{bp}$ of targeted exons. This represents 170 -fold enrichment of the $\mathrm{X}$ chromosome exons (Table 1). 1,462 SNPs were discovered on the X chromosome. Of these, 991 (68\%) were found to be previously annotated on dbSNP and 471 were novel SNPs (Table 2). For sample B1, there were $11,833,641$ unique sequences across the human genome. Of these, $23 \%$ of sequences were mapped to the $\mathrm{X}$ chromosome, $87 \%$ of these mapped on or within $500 \mathrm{bp}$ of targeted exons. This represents 85 -fold enrichment of the $\mathrm{X}$ chromosome exons (see Table 1). 2,385 SNPs were discovered on the $\mathrm{X}$ chromosome. Of these, 1,627 (68\%) were found to be previously annotated on dbSNP and 758 were novel SNPs (see Table 2). The discrepancy between the two individual samples within the duplex is attributable to quantification error. This technique is sensitive to quantification which can, in turn, affect the accuracy of equimolar pooling of multiplexed samples. We employed Nanodrop quantification to determine individual library concentrations however, PicoGreenbased quantification would be more effective at this critical point.

Pentaplex data (samples A3, B2, C1, D1 and E1) were split by index barcode and analysed independently. For individual samples, there was a median of 3,122,661 \pm 264,985 unique sequences across the human genome. Of these, $40 \pm 10 \%$ of sequences were mapped to the $\mathrm{X}$ chromosome, of these $91 \pm 0.5 \%$ of sequence reads map on or within $500 \mathrm{bp}$ of targeted exons. This represents an average 263 -fold enrichment of the $\mathrm{X}$ chromosome exons for each of the 5 individual samples in the pentaplex (Table 1). 2,102 \pm 281 SNPs were discovered on the $\mathrm{X}$ chromosome. Of these, $1,362 \pm 300$ were found to be previously annotated on dbSNP and $671 \pm 96$ were novel SNPs (Table 2). For sample A and B, which were genotyped three and two times, respectively, we found $2102 \pm 478$ and $2147 \pm 335$ SNPs, respectively.

We found that the overall depth of read across all targeted bases was comparable between the uniplex and pentaplex versions of individual A (Figure 1C). Although deeper read depth provides higher confidence in SNP calling, our read depth across all samples was $\sim 16 \mathrm{X}$, which is adequate for the GATK algorithm to confidently call variant genotypes in the sample at a confidence interval of 70 .

\section{Discussion and Conclusion}

Our work benchmarks the use of a new target enrichment technology combined with a barcode indexing method with the aim of reducing the time and costs associated with NGS platforms. We have demonstrated the ability to genotype five individuals across a $3.3 \mathrm{Mb}$ targeted region with confidence that we would detect the majority of the SNPs within the targeted region. Furthermore, we have shown that the sequencing depth obtained is adequate for credible SNP genotyping analysis at uniplex, duplex and pentaplex levels.

Our experiments describe re-sequencing of discontiguous exons where we observed that coverage per base was reduced at the flanks and increased at the middle of the sequenced regions (Figure 1B). Sequencing of contiguous regions, or larger blocks of discontiguous regions, would increase the average read depth across all samples. Additionally, with the introduction of high capacity sequencing technologies such as Illumina $\mathrm{Hi}$ $\mathrm{Seq}^{\mathrm{ma}}$, the read depth per multiplexed sample will increase dramatically providing higher confidence in SNP calling and the potential to increase plexity when read depth exceeds that required for confident genotyping. The successful demonstration of this methodology may facilitate the efficient identification of key genetic variants in disease linked loci.

\section{Methods}

\section{Ethics Statement}

All participants in this project provided informed consent for sample collection and retention. The project was approved by The Alfred Research and Ethics Unit (Approval number 312/10).

\section{Genomic DNA preparation and Library Construction}

Whole blood was taken from 5 individuals ( 3 males and 2 females) and genomic DNA was extracted using QIAamp DNA Blood Midi Kit (Qiagen, Hilden, Germany). Individual genomic DNA samples were quantified by Nanodrop (Agilent, California, USA) and 
equalised to $30 \mathrm{ng} / \mathrm{uL}$ and $100 \mathrm{uL}$ ( $3 \mathrm{ug}$ ) was sonically sheared to $200 \mathrm{bp}$ on the Covaris S2 system (Covaris, Massachusetts, USA) with the following parameters:

Duty Cycle: $10 \%$

Intensity: 5

Cycles per Burst: 200

Time (seconds): 180

Nucleotide overhangs produced as a result of the shearing process were converted to blunt ends using Klenow enzyme. The end-repair mix contained $27 \mathrm{uL}$ DNA sample, $48 \mathrm{uL}$ water, 10 uLT4 DNA Ligase buffer with $10 \mathrm{mM}$ ATP, $4 \mathrm{uL} 10 \mathrm{mM}$ dNTP mix, $5 \mathrm{uL}$ T4 DNA polymerase, $1 \mathrm{uL}$ Klenow enzyme and $5 \mathrm{uL}$ T4 PNK. Samples were incubated in a thermocycler at $20^{\circ} \mathrm{C}$ for 30 minutes. After incubation, samples were purified using QIAquick columns from a QIAquick PCR Purification Kit (Qiagen, Hilden, Germany) and eluted in 32 uL of Qiagen buffer EB.

Fragments were prepared for adapter ligation by addition of dATP to the 3' end of the blunt phosphorylated DNA fragments. A $50 \mathrm{ul}$ reaction mix was made for each sample containing $32 \mathrm{uL}$ DNA sample, 5 uL Klenow buffer, $10 \mathrm{uL} 1 \mathrm{mM}$ dATP and 3 uL Klenow fragment ( 3 ' to 5 ' exo minus). The reactions were incubated at $37^{\circ} \mathrm{C}$ for 30 minutes. After incubation, samples were purified using QIAquick MinElute columns from a MinElute PCR Purification Kit (Qiagen, Hilden, Germany) and eluted in $10 \mathrm{uL}$ of Qiagen buffer EB.

Index PE adapters (Multiplexing Sample Preparation Oligonucleotide Kit, PE-400-1001, Illumina, San Diego, USA) were ligated to the ends of the DNA fragments at a 10:1 molar ratio of adapter to insert DNA for all samples except A1. For sample A1, paired-end adapters (Paired End DNA Sample Preparation Kit, FC-102-1001, Illumina, San Diego, USA) was used at the same molar ratio as the other samples. A 50 ul reaction mix was made for each sample containing $10 \mathrm{uL}$ DNA sample, 25 uL 2X DNA ligase buffer, 10 uL Index PE adapter oligo mix and 5 uL DNA ligase. Samples were incubated in a thermocycler at $20^{\circ} \mathrm{C}$ for 15 minutes. After incubation, samples were purified using QIAquick columns from a QIAquick PCR Purification Kit (Qiagen, Hilden, Germany) and eluted in $30 \mathrm{uL}$ of Qiagen buffer EB.

Samples were individually purified on $2 \%$ HR agarose (Ambion, Texas, USA) gels in Tris-Acetate-EDTA buffer to remove unligated and self-ligated adapters and for size selection of products for cluster generation. A 100 bp DNA ladder (GeneWorks, Adelaide, Australia) was used for size selection. Agarose blocks corresponding to the 300-320 bp were extracted using an x-tracta device (LabGadget, Illinios, USA). Samples were purified using QIAquick Gel Extraction Kit (Qiagen, Hilden, Germany) and eluted in $30 \mathrm{uL}$ Qiagen buffer EB.
PCR was performed to selectively enrich each sample for DNA fragments with adapters ligated to both ends. Index barcodes are also introduced in this PCR. Two primers are used to amplify samples and a third unique indexing primer containing the indexing barcode is used to for samples discrimination after multiplex sequencing. A $50 \mathrm{uL}$ PCR reaction mix was produced containing 5 uL DNA, 25 uL Phusion DNA polymerase, $1 \mathrm{uL}$ PCR primer InPE1.0, $1 \mathrm{uL}$ PCR primer InPE2.0, $17 \mathrm{uL}$ water and $1 \mathrm{uL} \mathrm{PCR} \mathrm{index} \mathrm{primer.} \mathrm{Individual} \mathrm{sample}$ mixes contained sample specific PCR index primer. PCR was performed on a PTC-200 thermocycler (MJ Research, Massachusetts, USA) with the following cycle: 30 seconds at $98^{\circ} \mathrm{C}$, then 14 cycles of 10 seconds at $98^{\circ}$ C, 30 seconds at $65^{\circ} \mathrm{C}, 30$ seconds at $72^{\circ} \mathrm{C}$, followed by a final extension step of 5 minutes at $72^{\circ} \mathrm{C}$ and a hold step at $4^{\circ} \mathrm{C}$. Sample A1 was amplified using PE PCR Primers 1.0 and 2.0. Samples A2 - E1 were amplified using PCR Primer InPE 1.0 and 2.0 and PCR Index Primers $4-10$ respectively. After PCR, samples were purified using QIAquick columns from a QIAquick PCR Purification Kit (Qiagen, Hilden, Germany) and eluted in $50 \mathrm{uL}$ of Qiagen buffer EB.

The concentration of each library was determined by measuring absorbance and confirmed for downstream processing if the OD 260/280 was approximately 1.8 . After concentrations were determined, 1 ul of each library was analysed on 4-20\% acrylamide/TBE Novex gel (Invitrogen, California, USA) alongside a $100 \mathrm{bp}$ DNA ladder (GeneWorks, Adelaide, Australia) and visualised with SYBR Gold to confirm the size range of the library (see Figure 1E).

\section{Target Enrichment}

Genome partitioning was performed using SureSelect Target Enrichment Kit and SureSelect Human Chromosome X Exome Kit (Agilent, California, USA). Individual samples were pooled at equimolar ratios so the final pooled multiplex library concentration was $147 \mathrm{ng} / \mathrm{uL}$. A hybridisation buffer master mix was prepared for 3 library captures at room temperature containing $75 \mathrm{uL}$ SureSelect hybridisation buffer \#1, 3 uL SureSelect hybridisation buffer \#2, 30 uL SureSelect hybridisation buffer \#3 and 39 uL SureSelect hybridisation buffer \#4. $40 \mathrm{uL}$ of master mix was aliquoted into row A of a 96well PCR plate. $3.4 \mathrm{uL}$ of each library was aliquoted into row B. To each well of row B, 2.5 uL SureSelect Block \#1, 2.5 uL SureSelect Block \#2 and 0.6 uL SureSelect Block \#3 was added and mixed by pipetting. Wells were sealed with strip caps and samples were incubated on a thermocycler at $95^{\circ} \mathrm{C}$ for 5 minutes and $65^{\circ} \mathrm{C}$ for $5 \mathrm{~min}$ utes. An oligo capture library was prepared in PCR strip tubes by adding $5 \mathrm{uL}$ SureSelect Oligo Capture Library, $1 \mathrm{uL}$ nuclease-free water and $1 \mathrm{uL}$ RNase block to each 
of 3 tubes. The capture library was added to row $\mathrm{C}$ of the PCR plate, sealed with strip caps and the plate was incubated at $65^{\circ} \mathrm{C}$ for 2 minutes. After incubation, $13 \mathrm{uL}$ of hybridisation buffer mix was taken from row $\mathrm{A}$ and added to row $\mathrm{C}$ and $7 \mathrm{uL}$ of prepared library from row $B$ was added to row $C$. The contents of row $C$ were mixed by pipetting and the plate was sealed with plate sealing film. The hybridisation mixture was incubated at $65^{\circ} \mathrm{C}$ for 48 hours.

Dynabead M-280 Streptavidin (Invitrogen, California, USA) was prepared by vortexing and adding 50 ul Dynabeads to a $1.5 \mathrm{~mL}$ microfuge tube for each capture library. Dynabeads were washed 3 times by adding $200 \mathrm{uL}$ SureSelect binding buffer, mixing on a vortex for 5 seconds, applying tube contents to magnetic separation and removing supernatant. After 3 washes, beads were resuspended in $200 \mathrm{uL}$ SureSelect binding buffer. Each hybridisation mixture was added to a Dynabead solution after hybridisation and each tube was mixed by inverting 5 times. Each hybrid capture/bead solution was incubated at room temperature on a nutator for 30 minutes. Beads and buffer were then separated by magnetic separation and the supernatant was removed. Beads were resuspended in $500 \mathrm{uL}$ SureSelect wash buffer \#1 by vortex mixing for 5 seconds and incubated for 15 minutes at room temperature. Beads were washed 3 times by applying tube contents to magnetic separation, removing supernatant, adding $500 \mathrm{uL}$ SureSelect wash buffer \#2 (prewarmed to $65^{\circ} \mathrm{C}$ ), mixing on a vortex for 5 seconds, incubating samples at $65^{\circ} \mathrm{C}$ for 10 minutes and inverting tube to mix. After 3 washes, beads were resuspended in $50 \mathrm{uL}$ SureSelect elution buffer and mixed by vortexing for 5 seconds. All samples were incubated for 10 minutes at room temperature. Beads and buffer were then separated by magnetic separation and the buffer was moved to a new $1.5 \mathrm{~mL}$ microfuge tube. $50 \mathrm{uL}$ SureSelect neutralisation buffer was added to the buffer.

Capture solution desalting was performed using a QIAquick MinElute PCR purification column from a MinElute PCR Purification Kit (Qiagen, Hilden, Germany). MinElute columns were brought to room temperature. $500 \mathrm{uL}$ PBI was added to the sample solution and mixed by pipetting. Columns were placed in a $2 \mathrm{~mL}$ collection tube and $600 \mathrm{uL}$ sample was added to the column. Samples were spun at 13,000 rpm for 60 seconds and flow-through was discarded. $750 \mathrm{uL}$ of buffer PE was added to the column, samples were spun at 13,000 rpm for 60 seconds and flow-through was discarded. Column was spun again at 13,000 rpm for 60 seconds and column was placed in a new $1.5 \mathrm{~mL}$ microfuge tube. 15 uL buffer EB was applied directly to the MinElute filter and allowed to incubate for 60 seconds.
Samples were spun at 13,000 rpm for 60 seconds and collected eluate (captured library) was stored at $-20^{\circ} \mathrm{C}$.

A post-hybridisation PCR was performed to amplify the index adapter ligated, genome partitioned samples. For each capture solution, an amplification reaction was performed using Phusion High Fidelity DNA Polymerase (Finnzymes, Espoo, Finland). The reaction mix contained $10 \mathrm{uL}$ 5X Phusion High Fidelity buffer, $1.5 \mathrm{uL}$ 10 mM dNTP mix, 1 uL 10 uM P5 primer, 1 uL 10 uM P7 primer, 1 unit Phusion DNA polymerase, $1 \mathrm{uL}$ of captured DNA and $35 \mathrm{uL}$ nuclease-free water. A PCR was performed on a PTC-200 thermocycler (MJ Research, Massachusetts, USA) with the following cycle: 30 seconds at $98^{\circ} \mathrm{C}$, then 18 cycles of 10 seconds at $98^{\circ} \mathrm{C}, 30$ seconds at $57^{\circ} \mathrm{C}, 30$ seconds at $72^{\circ} \mathrm{C}$, followed by a final extension step of 5 minutes at $72^{\circ} \mathrm{C}$ and a hold step at $4^{\circ} \mathrm{C}$. Libraries were purified using QIAquick columns from a QIAquick MinElute Kit (Qiagen, Hilden, Germany) and eluted in $15 \mathrm{uL}$ of Qiagen buffer EB and Tween20 (Sigma-Aldrich, Missouri, USA) was added to a final concentration of $0.1 \% .1 \mathrm{uL}$ of each library was analysed on 4-20\% acrylamide/TBE Novex gel (Invitrogen, California, USA) alongside a $100 \mathrm{bp}$ DNA ladder (GeneWorks, Adelaide, Australia) and visualised with Ethidium Bromide to confirm the size range and amplification of each library. Final library concentration measured by PicoGreen-based assay (Quant-iT PicoGreen dsDNA Assay Kit, Invitrogen) vs lambda DNA standard (Table 3).

\section{Sequence Generation}

Libraries were diluted to $10 \mathrm{nM}$ concentrations before further dilution to $4.5 \mathrm{pM}$ for cluster generation and sequencing-by-synthesis on the Illumina Genome Analyser II (running SCS2.3/IPAR). Each library was sequenced on a single lane of an 8-lane flow-cell. GAII Images were processed using the Illumina Pipeline (v 1.3.2). Images were processed through Firecrest, producing signal intensity files. These signal intensities were processed by Bustard into base calls and ultimately sequence files. The uniplex had quality issues in the last 7 base pairs which prevented alignment against the reference genome. These problematic bases were trimmed off using the the fastx toolkit [13]. This caused the length of the uniplex sequences to drop from $64 \mathrm{bp}$

Table 3 Concentrations of final multiplexed libraries for sequencing

\begin{tabular}{ccc}
\hline Library Number & Concentration $(\mathbf{n g} / \mathbf{u L})$ & Calculated $\mathbf{n M}^{*}$ \\
\hline 1 & 12.7 & 57 \\
2 & 11.8 & 53 \\
3 & 5.8 & 26 \\
\hline
\end{tabular}

*Calculation based on $340 \mathrm{bp}$ average size and MW of one base pair $=660 \mathrm{~g} /$ mol. 
to $57 \mathrm{bp}$ long. The multiplexed samples were required to be split into separate sequence files for each sample, and the bar code sequence needed to be removed. This was done using the fastx toolkit [13], with a maximum bar code mismatch (edit distance) score of 2 .

\section{Alignment and SNP calling}

The sequence files were aligned against the hg18 reference genome using the BWA (v 0.5.5) aligner, using default parameters [11]. The resulting SAM files were sorted and compressed into BAM files by using the MergeSamFiles tool which is part of the Picard toolkit [12]. An index file for each BAM file was generated using the SAMTools index function [12]. The GATK Unified Genotyper tool was used to generate a list of SNPs for each sample at different confidence levels (Figure 1D). These lists of SNPs where matched against dbSNP (build 130) [14] via Galaxy's Genomic Intervals tool kit [15]. This gave a list of identified SNPs which were known to dbSNP.

\section{Enrichment Analysis}

The enrichment level of the targeted region was calculated by using the SAMTools pileup tool [12] to generate a count of reads on each individual base pair, and only select values which fell in the regions defined by the SureSelect design. The generated values were analysed in R [16] to generate the box plot (Figure 1C) and associated statistics. The fold enrichment factor was calculated by dividing the average base coverage value within the targeted region by the average base coverage value outside of the targeted region (background genomic reads). Average base coverage was the average number of times a base was read within the designated interval.

\section{Adapter and Primer Sequences}

PE Genomic Adapters (Illumina, California, USA)

5' P-GATCGGAAGAGCGGTTCAGCAGGAATGCCGAG

5' ACACTCTTTCCCTACACGACGCTCTTCCGATCT

A1)

PE PCR Primers (used to amplify non-indexed sample

PE PCR Primer 1.0 (Illumina, California, USA)

5'AATGATACGGCGACCACCGAGATCTACACTC-

TTTCCCTACACGACGCTCTTCCGATCT

PE PCR Primer 2.0 (Illumina, California, USA)

5'CAAGCAGAAGACGGCATACGAGATCGGTCTC-

GGCATTCCTGCTGAACCGCTCTTCCGATCT

Multiplexing Adapters (Illumina, California, USA)

5' P-GATCGGAAGAGCACACGTCT

5' ACACTCTTTCCCTACACGACGCTCTTCCGATCT
Multiplexing PCR Primers (Illumina, California, USA)

Multiplexing PCR Primer 1.0

5'AATGATACGGCGACCACCGAGATCTACACTC-

TTTCCCTACACGACGCTCTTCCGATCT

Multiplexing PCR Primer 2.0

5' GTGACTGGAGTTCAGACGTGTGCTCTTCCG-

\section{ATCT}

PCR Indexing Primers (Illumina, California, USA)

PCR Primer Index 4: 5'CAAGCAGAAGACGG-

CATACGAGATTGGTCAGTGACTGGAGTTC

PCR Primer Index 5: 5'CAAGCAGAAGACGGC-

ATACGAGATCACTGTGTGACTGGAGTTC

PCR Primer Index 6: 5'CAAGCAGAAGACGGCAT-

ACGAGATATTGGCGTGACTGGAGTTC

PCR Primer Index 7: 5'CAAGCAGAAGACGGCA-

TACGAGATGATCTGGTGACTGGAGTTC

PCR Primer Index 8: 5'CAAGCAGAAGACGGCA-

TACGAGATTCAAGTGTGACTGGAGTTC

PCR Primer Index 9: 5'CAAGCAGAAGACGG-

CATACGAGATCTGATCGTGACTGGAGTTC

PCR Primer Index 10: 5'CAAGCAGAAGACG-

GCATACGAGATAAGCTAGTGACTGGAGTTC

Illumina oligonucleotide sequences ${ }^{\odot}$ 2006-2008 Illu-

mina, Inc. All rights reserved.

Flanking Post-Capture PCR Primers (GeneWorks, South Australia, Australia)

P5 Primer 5'-AATGATACGGCGACCACCG

P7 Primer 5'-CAAGCAGAAGACGGCATACGA

\section{Funding}

This work was supported by BakerIDI Heart and Diabetes Institute.

\section{Acknowledgements}

The authors wish to extend their gratitude toward Assam El-Osta for contributing to the support for this project.

\section{Author details \\ ${ }^{1}$ Genomics and Systems Biology, Baker IDI Heart and Diabetes Institute, Melbourne, Australia. ${ }^{2}$ Systems Integrations, Baker IDI Heart and Diabetes Institute, Melbourne, Australia. ${ }^{3}$ Human Epigenetics, Baker IDI Heart and Diabetes Institute, Melbourne, Australia. ${ }^{4}$ DNA and Blood Profiling Facility, Baker IDI Heart and Diabetes Institute, Melbourne, Australia. ${ }^{5} \mathrm{GeneW}$ orks Pty Ltd, Adelaide, Australia. ${ }^{6}$ Department of Biochemistry, School of Medicine, University of Melbourne, Melbourne, Australia. ${ }^{7}$ Metastasis Research Laboratory, Peter MacCallum Cancer Centre, Melbourne, Australia.}

\section{Authors' contributions}

$\mathrm{NC}, \mathrm{IH}$ and $\mathrm{J}$ conceived of and designed the study. NC, RK and AR undertook the molecular genetic analysis. NC, AK, SL, IH and JJ analysed the sequence data. NC and JJ wrote the manuscript. All authors read, provided comments on and approved the manuscript.

\section{Competing interests}

The authors declare that they have no competing interests.

Received: 25 March 2010 Accepted: 18 November 2010 Published: 18 November 2010 


\section{References}

1. Craig DW, Pearson JV, Szelinger S, Sekar A, Redman M, Corneveaux JJ, Pawlowski TL, Laub T, Nunn G, Stephan DA, et al: Identification of genetic variants using bar-coded multiplexed sequencing. Nat Methods 2008, 5(10):887-893.

2. Porreca GJ, Zhang K, Li JB, Xie B, Austin D, Vassallo SL, LeProust EM, Peck BJ, Emig CJ, Dahl F, et al: Multiplex amplification of large sets of human exons. Nat Methods 2007, 4(11):931-936.

3. Turner EH, Lee C, Ng SB, Nickerson DA, Shendure J: Massively parallel exon capture and library-free resequencing across 16 genomes. Nat Methods 2009, 6(5):315-316

4. Gnirke A, Melnikov A, Maguire J, Rogov P, LeProust EM, Brockman W, Fennell T, Giannoukos G, Fisher S, Russ $C$, et al: Solution hybrid selection with ultra-long oligonucleotides for massively parallel targeted sequencing. Nat Biotechnol 2009, 27(2):182-189.

5. Hodges E, Xuan Z, Balija V, Kramer M, Molla MN, Smith SW, Middle CM, Rodesch MJ, Albert TJ, Hannon GJ, et al: Genome-wide in situ exon capture for selective resequencing. Nat Genet 2007, 39(12):1522-1527.

6. Daines B, Wang H, Li Y, Han Y, Gibbs R, Chen R: High-throughput multiplex sequencing to discover copy number variants in Drosophila. Genetics 2009, 182(4):935-941.

7. Lefrancois P, Euskirchen GM, Auerbach RK, Rozowsky J, Gibson T, Yellman CM, Gerstein M, Snyder M: Efficient yeast ChIP-Seq using multiplex short-read DNA sequencing. BMC Genomics 2009, 10:37.

8. Cronn R, Liston A, Parks M, Gernandt DS, Shen R, Mockler T: Multiplex sequencing of plant chloroplast genomes using Solexa sequencing-bysynthesis technology. Nucleic Acids Res 2008, 36(19):e122.

9. Smith AM, Heisler LE, Mellor J, Kaper F, Thompson MJ, Chee M, Roth FP, Giaever G, Nislow C: Quantitative phenotyping via deep barcode sequencing. Genome Res 2009, 19(10):1836-1842.

10. Mir KU: Sequencing genomes: from individuals to populations. Brief Funct Genomic Proteomic 2009, 8(5):367-378.

11. Li H, Durbin R: Fast and accurate short read alignment with BurrowsWheeler transform. Bioinformatics 2009, 25(14):1754-1760.

12. Li H, Handsaker B, Wysoker A, Fennell T, Ruan J, Homer N, Marth G, Abecasis G, Durbin R: The Sequence Alignment/Map format and SAMtools. Bioinformatics 2009, 25(16):2078-2079.

13. Pearson WR, Wood T, Zhang Z, Miller W: Comparison of DNA sequences with protein sequences. Genomics 1997, 46(1):24-36

14. Wheeler DL, Chappey C, Lash AE, Leipe DD, Madden TL, Schuler GD, Tatusova TA, Rapp BA: Database resources of the National Center for Biotechnology Information. Nucleic Acids Res 2000, 28(1):10-14.

15. Taylor J, Schenck I, Blankenberg D, Nekrutenko A: Using galaxy to perform large-scale interactive data analyses. Curr Protoc Bioinformatics 2007, Chapter 10, Unit 1015

16. Team RDC: R: A language and environment for statistical computing. Computing RFfS. Vienna, Austria: R Foundation for Statistical Computing: 2009.

doi:10.1186/1471-2164-11-641

Cite this article as: Cummings et al:: Combining target enrichment with barcode multiplexing for high throughput SNP discovery. BMC Genomics 2010 11:641.

\section{Submit your next manuscript to BioMed Central and take full advantage of:}

- Convenient online submission

- Thorough peer review

- No space constraints or color figure charges

- Immediate publication on acceptance

- Inclusion in PubMed, CAS, Scopus and Google Scholar

- Research which is freely available for redistribution

Submit your manuscript at www.biomedcentral.com/submit
Ciomed Central 